\title{
Erratum to: A modified serial blood sampling technique and utility of dried-blood spot technique in estimation of blood concentration: application in mouse pharmacokinetics
}

\author{
Vishwanath Kurawattimath • Krishna Pocha • \\ T. Thanga Mariappan • Ravi Kumar Trivedi • \\ Sandhya Mandlekar
}

Published online: 27 October 2011

(C) Springer-Verlag France 2011

\section{Erratum to: Eur J Drug Metab Pharmacokinet}

DOI 10.1007/s13318-011-0066-5

Unfortunately the original version has been published with an incomplete Acknowledgment section.

The complete section should read:

Acknowledgments We would like to thank Shahe Mahammad, Ajay Saxena and Abhijith Rao for their help in making formulations and bioanalysis. We also gratefully acknowledge the contribution and guidance provided by Celia D'Arienzo, Bristol-Myers Squibb, Princeton, NJ, US, towards establishing the DBS technique in our laboratory. Also acknowledged is the following article published by D'Arienzo et al.: D'Arienzo CJ, Ji QC, Discenza L, Cornelius G, Hynes J, Cornelius L, Santella JB, Olah T (2010) DBS sampling can be used to stabilize prodrugs in drug discovery rodent studies without the addition of esterase inhibitors. Bioanalysis 8:1415-1422.

The online version of the original article can be found under doi:10.1007/s13318-011-0066-5.

V. Kurawattimath $\cdot$ K. Pocha $\cdot$ T. Thanga Mariappan . R. K. Trivedi $\cdot$ S. Mandlekar $(\bowtie)$

Pharmaceutical Candidate Optimization, Biocon Bristol Myers-Squibb R\&D Centre (BBRC), Syngene International Limited, Biocon Park, Plot 2 and 3, Bommasandra IV Phase, Bangalore 560 099, India

e-mail: sandhya.mandlekar@bms.com 